\title{
An Explanation for Reflex Blink Hyperexcitability in Parkinson's Disease. II. Nucleus Raphe Magnus
}

\author{
Michele A. Basso ${ }^{1}$ and Craig Evinger ${ }^{2}$ \\ ${ }^{1}$ Department of Psychology, and 2Departments of Neurobiology and Behavior and Ophthalmology, SUNY Stony Brook, \\ Stony Brook, New York 11794-5230
}

Hyperexcitable reflex blinks are a cardinal sign of Parkinson's disease. The first step in the circuit linking the basal ganglia and brainstem reflex blink circuits is the inhibitory nigrostriatal pathway (Basso et al., 1996). The current study reports the circuits linking the superior colliculus (SC) to trigeminal reflex blink circuits. Microstimulation of the deep layers of the SC suppresses subsequent reflex blinks at a latency of $5.4 \mathrm{msec}$. This microstimulation does not activate periaqueductal gray antinociceptive circuits. The brainstem structure linking SC to reflex blink circuits must suppress reflex blinks at a shorter latency than the SC and produce the same effect on reflex blink circuits as SC stimulation, and removal of the structure must block SC modulation of reflex blinks. Only the nucleus raphe magnus
(NRM) meets these requirements. NRM microstimulation suppresses reflex blinks with a latency of $4.4 \mathrm{msec}$. Like SC stimulation, NRM microstimulation reduces the responsiveness of the spinal trigeminal nucleus. Finally, blocking the receptors for the NRM transmitter serotonin eliminates SC modulation of reflex blinks, and muscimol inactivation of the NRM transiently prevents SC modulation of reflex blinks. Thus, the circuit through which the basal ganglia modulates reflex blinking is (1) the substantia nigra pars reticulata inhibits SC neurons, (2) the SC excites tonically active NRM neurons, and (3) NRM neurons inhibit spinal trigeminal neurons involved in reflex blink circuits.

Key words: Parkinson's disease; blink reflex; superior colliculus; trigeminal complex; rats
Reduced dopaminergic tone in the basal ganglia (BG) produces marked reflex blink hyperexcitability in animals (Shallert et al., 1989; Basso et al., 1993) including humans (Kimura, 1973a). The companion paper [Basso et al., 1996 (this issue)] demonstrates that the superior colliculus (SC) mediates this increase in reflex blink excitability. The present experiments identify the neuronal linkage between the SC and the reflex blink circuitry within the brainstem.

The rodent SC has two major descending projections. First, the ipsilateral descending pathway originates from the entire rostral caudal extent of the medial SC. The axons forming this pathway terminate primarily in the parabigeminal nucleus, cuneiform nucleus, ventrolateral midbrain pontine reticular formation, pontine nuclei, and the pontomedullary reticular formation (Redgrave et al., 1987, 1988). Stimulation of this projection system elicits defensive or withdrawal behaviors (Dean et al., 1988a,b, 1989; Mitchell et al., 1988; Redgrave et al., 1988). The second descending pathway, the contralateral tecto-reticulospinal tract, originates primarily from the lateral SC (Dean et al., 1986; Redgrave et al., 1987; Grantyn, 1988). These axons form the predorsal bundle, (May and Hall, 1984, 1986) and terminate in the nucleus reticularis tegmenti pontis, pedunculopontine or parabrachial area, caudal pontine reticular nuclei, pontomedullary reticular formation, pontine raphe, and the ventral spinal cord (Redgrave et al., 1987). Stimulation of the lateral SC elicits orienting behaviors

\footnotetext{
Received March 18, 1996; revised July 31, 1996; accepted Aug. 27, 1996.

This work was supported by National Eye Institute Grant EY07391 (C.E.) and summer fellowships from the Parkinson's Disease Foundation. We thank Donna Schmidt for her expert technical assistance.

Correspondence should be addressed to Craig Evinger, Department of Neurobiology and Behavior, SUNY Stony Brook, Stony Brook, NY 11794-5230.

Dr. Basso's current address: Laboratory of Sensorimotor Research, National Eye Institute, Building 49, Room 2A50, Bethesda, MD 20892-4435.

Copyright (C) 1996 Society for Neuroscience $0270-6474 / 96 / 167318-13 \$ 05.00 / 0$
}

(Dean, 1988a) and suppresses reflex blinks in rodents (Basso et al., 1996) and primates (Lu et al., 1993).

Many of the brainstem areas that receive lateral SC afferents could mediate SC modulation of reflex blinks. Because SC inactivation increases reflex blink excitability and SC activation decreases reflex blink excitability, the SC must excite a tonically active neuron that inhibits the reflex blink circuit. The inhibitory, midline omnipause neurons (OPNs) might fill this role. Tonically active OPNs receive an excitatory input from the rostral SC (Raybourn and Keller, 1977; Paré and Guitton, 1994) and cease discharging with both saccadic eye movements (Evinger et al., 1982; Paré and Guitton, 1994) and reflex blinks (Fuchs et al., 1991; Mays and Morrisse, 1994). Microstimulation in the OPN region suppresses reflex blinks in rats (C. Evinger, unpublished observations) and monkeys (Mays and Morrisse, 1994). Nevertheless, OPNs are unlikely to link the SC to reflex blink circuits. First, although microstimulation of the monkey SC suppresses reflex blinks (Lu et al., 1994; Gnadt et al., in press), the fixation zone of the superior colliculus that activates OPNs (Raybourn and Keller, 1977; Paré, 1994) is not the lowest threshold point for suppressing reflex blinks. Second, if reflex blink hyperexcitability of Parkinson's disease occurred because increased nigral inhibition of the fixation zone reduced the excitatory drive to OPNs, Parkinson's disease could produce uncontrollable saccadic eye movements. Clinical evidence shows that patients with Parkinson's disease actually exhibit an increased saccadic latency (Bronstein et al., 1985; Rascol et al., 1989; White et al., 1989; Lueck et al., 1990; Müller et al., 1994). Thus, distinct populations of SC neurons appear to control saccadic eye movements and reflex blink excitability. The tonically active ponto-medullary raphe nuclei are the most likely candidates to mediate SC modulation of reflex blinking. Our data demonstrate that the serotonergic neurons of the ponto-medullary nucleus raphe magnus (NRM) link the BG to 


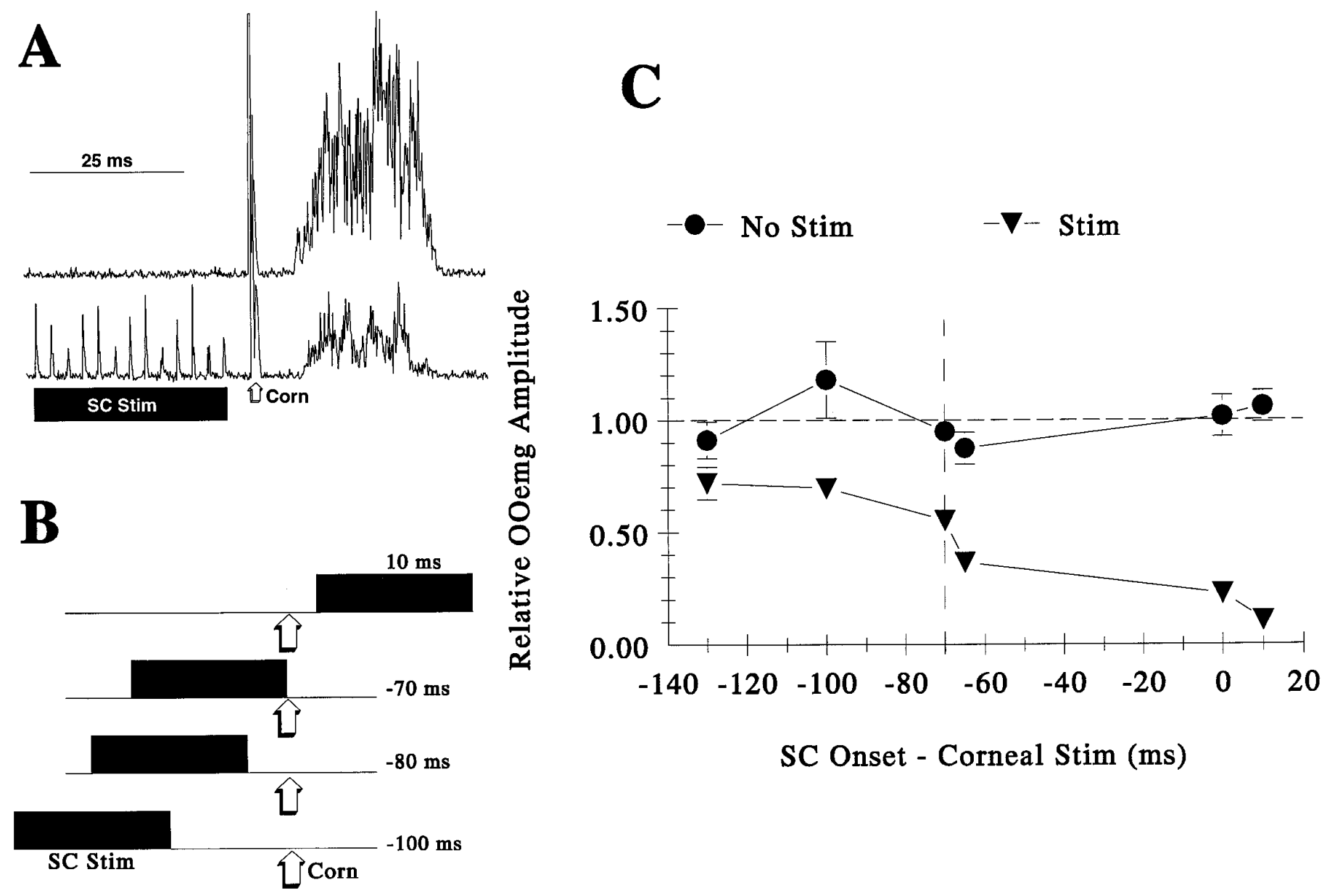

Figure 1. Effect of the delay between the onset of the SC stimulation and the occurrence of the corneal stimulus on reflex blink magnitude. $A$, OOemg response to a corneal stimulus (Corn) with (bottom trace) and without (top trace) a preceding $40 \mu \mathrm{A}, 70 \mathrm{msec}, 200 \mathrm{~Hz}$ train of SC stimulation (black bar) that terminated $5 \mathrm{msec}$ before the corneal stimulus. Each trace is the average of five rectified blinks. $B$, The diagram illustrates stimulus conditions. The corneal stimulus (Corn) occurred $100 \mathrm{msec}(-100 \mathrm{msec}), 80 \mathrm{msec}(-80 \mathrm{msec}), 70 \mathrm{msec}(-70 \mathrm{msec})$ after the onset of a $70 \mathrm{msec}$ train of SC stimulation (SC Stim) or $10 \mathrm{msec}(10 \mathrm{msec})$ before the onset of SC stimulation. $C$, Effect of SC stimulation on reflex blink magnitude as a function of time between corneal stimulation and the onset of a $70 \mathrm{msec}$ train of SC stimulation $(\boldsymbol{\nabla})$ compared to trials without superior colliculus stimulation $(\bullet)$. All data are normalized to the mean magnitude of all blinks at all delays without SC stimulation. Each point is the mean of 25 blinks (5 blinks from 5 animals), and the error bars are SEM.

the reflex blink circuit through the SC. Microstimulation of the NRM suppresses reflex blinks $\sim 1$ msec faster than does the SC. Blocking serotonin receptors prevents SC suppression of reflex blinks. Finally, temporary inactivation of the NRM eliminates SC suppression of the reflex blinks.

\section{MATERIALS AND METHODS}

Subjects. Male Sprague Dawley rats weighing between 150 and $400 \mathrm{gm}$ served as subjects. Animals were maintained on a 12 hour light/dark cycle and fed ad libitum. All procedures strictly adhered to federal, state, and university guidelines concerning the use of animals in research.

Acute preparation. Animals were prepared as described in the companion paper [Basso et al., 1996 (this issue)]. Briefly, rats were sedated with xylazine and anesthetized with urethane. A pair of silver ball electrodes were placed on the cornea to evoke blinks, and electrodes were implanted into the lateral and medial margins of the orbicularis oculi (OO) muscle to record its electromyographic activity (OOemg). Corneal stimulation parameters for each animal were determined by adjusting the intensity and duration of the electrical pulse to the cornea to evoke a consistent OOemg response, with an intertrial interval of $50 \pm 5 \mathrm{sec}$. The stimulation parameters remained constant for each animal throughout the testing session. In two rats, a nerve cuff was placed around the zygomatic branch of the facial nerve to activate facial motoneurons antidromically.
Electrical stimulation of this nerve produced twitches of the OO and upper vibrissal pad.

Microstimulation. Glass microelectrodes filled with $2 \mathrm{M}$ sodium acetate saturated with fast green were used for stimulation. To activate neural structures, a $70 \mathrm{msec}$ train of $200 \mathrm{~Hz}, 80 \mu \mathrm{sec}$ duration stimuli was delivered $75 \mathrm{msec}$ before corneal stimulation. The stimulus intensities ranged from 10 to $50 \mu \mathrm{A}$. To establish the latency of stimulation effects, a single $80 \mu \mathrm{sec}$ stimulus was presented $0,10,20$, or $30 \mathrm{msec}$ after the corneal stimulus. The stimulus intensities for the single stimuli ranged from 50 to $120 \mu \mathrm{A}$. In all experiments, stimulation and nonstimulation trials were alternated with an intertrial interval of $50 \pm 5 \mathrm{sec}$.

Drug protocols. We examined the effects of muscimol, a GABA agonist, metergoline, a serotonin blocker, and naloxone, an opioid antagonist, on SC modulation of reflex blinks. Muscimol (1\%) dissolved in saline was injected into brain structures to decrease neural activity. In one animal, $0.5 \mu \mathrm{l}$ of muscimol was injected through a 30 -gauge syringe into the SC while recording spinal trigeminal field potentials and OOemg activity evoked by corneal stimulation. In the other experiments, 50-100 $\mathrm{nl}$ of muscimol was pressure-injected with a Picospritzer (General Valve, Fairfield, NJ) through one barrel of a double-barrel recording electrode. The other barrel of the microelectrode contained $2 \mathrm{~m}$ sodium acetate saturated with fast green and was used to establish the level of reflex blink suppression produced by microstimulation. The electrode pair was placed stereotaxically into the NRM. The electrode position was marked by 


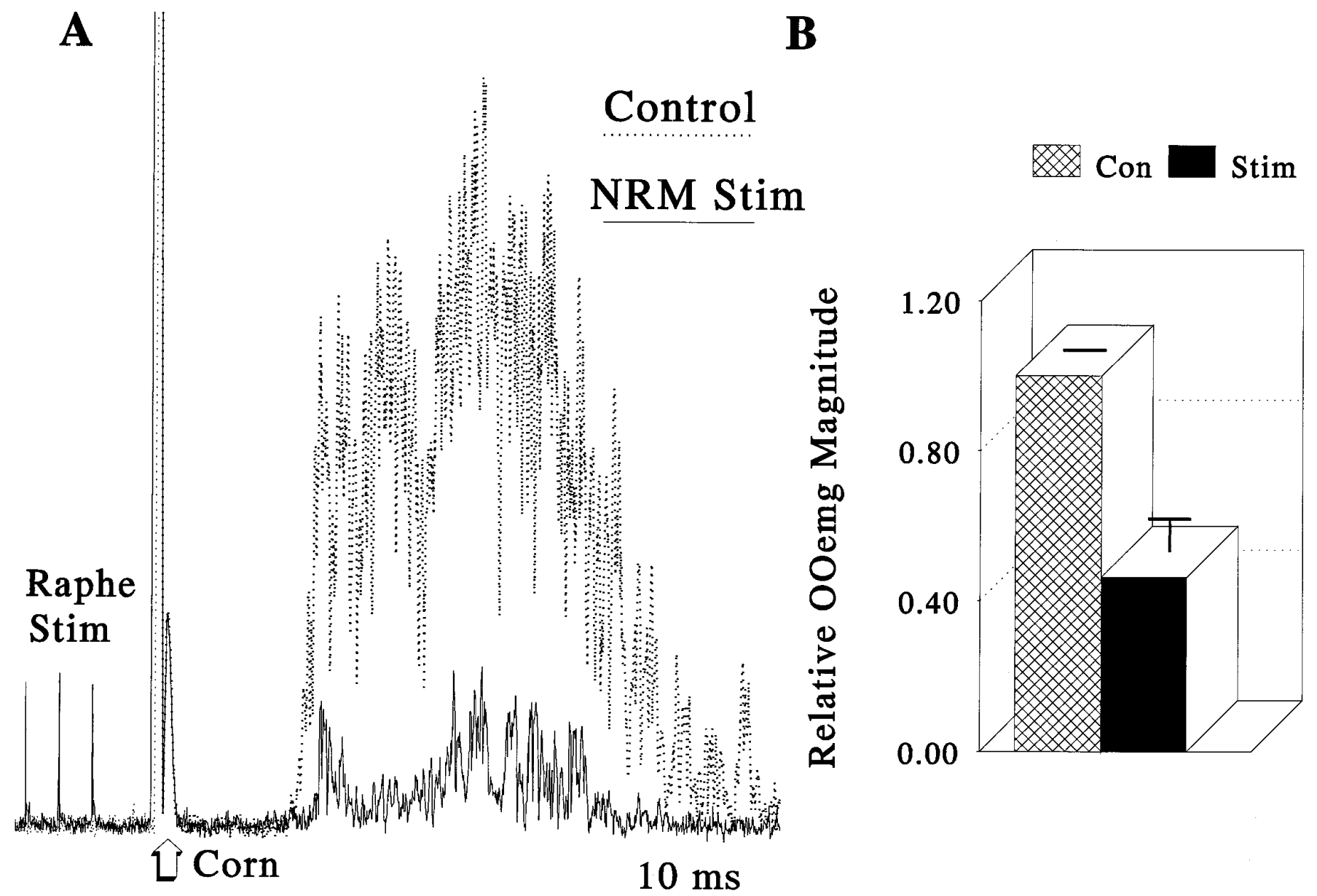

Figure 2. Suppression of reflex blinks by NRM microstimulation. A, Orbicularis oculi response to a corneal stimulus (Corn) with (solid line) and without (dotted line) a preceding $70 \mathrm{msec}, 200 \mathrm{~Hz}, 15 \mu \mathrm{A}$ stimulus train to the nucleus raphe magnus (Raphe Stim) that ended 5 msec before the corneal stimulus. Each trace is the average of five rectified responses. $B$, Group data from four animals (at least 5 blinks per condition per animal) illustrating OOemg magnitude with preceding NRM microstimulation (solid bar, Stim) relative to OOemg blink magnitude without NRM stimulation (hatched bar, Con). Error bars are SEM.

passing current through the fast green barrel (Thomas and Wilson, 1965). To block a broad range of serotonin receptor types, $6 \mathrm{mg} / \mathrm{kg}$ metergoline diluted in 5.0\% ascorbic acid was injected subcutaneously (Middlemiss and Trickleybank, 1992) while recording trigeminal field potentials and OOemg activity evoked by corneal stimulation. To test opioid mechanisms in reflex blink suppression, $2 \mathrm{mg} / \mathrm{kg}$ naloxone diluted in saline was injected subcutaneously in two animals while measuring the amount of reflex blink suppression produced by microstimulation of the SC.

Data acquisition and analysis. OOemg signals were acquired and stored on a computer $(4000 \mathrm{~Hz}, 12$-bit A/D resolution) and analyzed off-line using an interactive computer program that integrated OOemg records and determined latencies. When acquiring trigeminal field data and OOemg data simultaneously, both records were acquired and stored on a computer at $15 \mathrm{kHz}$ per channel (12-bit A/D resolution).

Statistical procedures. OOemg and field data were analyzed separately for each experimental manipulation. OOemg data were normalized to the mean blink amplitude for all animals, and nonparametric statistics were used to compare reflex blink amplitude with and without stimulation trains. Analyses assessing drug effects compared the amount of reflex blink suppression with and without SC stimulation before and after the drug using parametric statistics. To determine the latency of reflex blink suppression after a single stimulus, five consecutive OOemg responses of either stimulated or unstimulated trials were collected at $15 \mathrm{kHz}$ and averaged. We compared the two averaged wave forms by subtracting the OOemg waveform with a stimulus from the OOemg wave form without a stimulus. We took the mean and SD of the differences of $3 \mathrm{msec}$ of baseline data. We defined the suppression evoked by microstimulation as significant when the difference between the waveforms was 2 SD below the mean of the differences in the baseline for $>30$ consecutive points $(2$ msec). We calculated latency as the time after the stimulus when the stimulated wave form first showed the 2 SD decrease in magnitude that lasted at least 30 consecutive points. The $2 \mathrm{msec}$ period ensured that short random differences between the two wave forms were ignored. After analysis, the wave forms were smoothed with a $250 \mathrm{~Hz}$ low pass filter to facilitate visual comparison of the records.

Histology. At the end of the experiments, deeply anesthetized animals were perfused intracardially with a warm solution of $6.0 \%$ dextran in 0.1 $\mathrm{M}$ phosphate buffer ( $\mathrm{PB} ; \mathrm{pH} 7.4)$ and then with cold $10 \%$ formalin in 0.1 M PB. The brains were then immersed in a $30 \%$ sucrose solution in PB. The brains were cut into $100 \mu \mathrm{m}$ frozen sections and stained with cresyl violet to reconstruct electrode placement sites as marked by fast green dye injections.

\section{RESULTS}

Because suppression of reflex blinks was most effective at deep SC stimulation sites, in the initial experiments we investigated whether SC stimulation suppressed reflex blinks by directly activating periaqueductal grey (PAG) antinociceptive circuits. If blink suppression involved PAG mechanisms, then the effects of SC stimulation should be opioid-sensitive and last for periods of minutes (Levine et al., 1991). Stimulation of the cornea in five anesthetized rats evoked a single-component burst of OOemg activity that could be suppressed by a 70 -msec-duration train of 


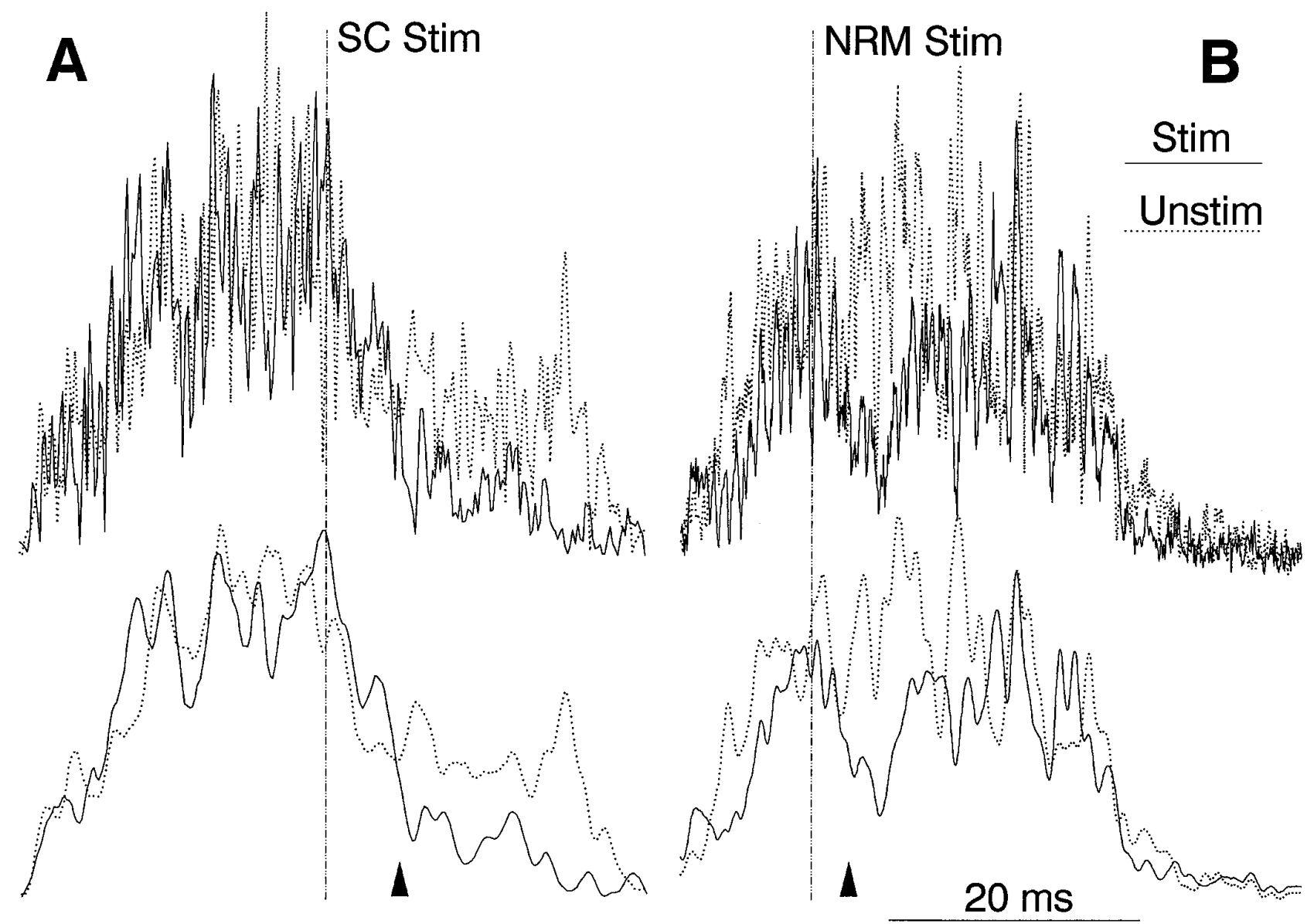

Figure 3. A single stimulus to the superior colliculus or the nucleus raphe magnus transiently decreases reflex blink amplitude. $A$, Unfiltered OOemg response to corneal stimulation with (solid line, SC Stim) and without (dotted line, Unstim) a single $80 \mu$ sec pulse to the superior colliculus (SC Stim, dashed vertical line). Traces are unfiltered (top traces) or filtered (bottom traces) at $250 \mathrm{~Hz}$ to facilitate comparison of the stimulated and unstimulated records. Each trace is the average of five rectified OOemg responses. $B$, A single stimulus of the nucleus raphe magnus transiently decreases blink amplitude at a shorter latency than SC stimulation. OOemg response to corneal stimulation with (solid line, NRM Stim) and without (dotted line, Unstim) a single 80 $\mu \mathrm{sec}$ pulse to the nucleus raphe magnus (NRM Stim, dashed vertical line). Traces are unfiltered (top traces) or filtered (bottom traces) at $250 \mathrm{~Hz}$ to facilitate comparison of the stimulated and unstimulated records. Each trace is the average of five rectified OOemg responses.

\begin{tabular}{|c|c|c|}
\hline $\begin{array}{l}\text { Latency from } \\
\text { OOemg onset (msec) }\end{array}$ & $\begin{array}{l}\text { Superior } \\
\text { colliculus stim }\end{array}$ & $\begin{array}{l}\text { Nucleus raphe } \\
\text { magnus stim }\end{array}$ \\
\hline 13 & & 4.1 \\
\hline 18 & & 5.9 \\
\hline 12.7 & & 3.5 \\
\hline 26.2 & & 4 \\
\hline 16.8 & 5.75 & \\
\hline 15.8 & 5 & \\
\hline
\end{tabular}

Latency of the decrease in OOemg magnitude after a single stimulus to the superior colliculus (Superior colliculus stim) or the nucleus raphe magnus (Nucleus raphe magnus stim) as a function of the time after the onset of corneally evoked OOemg activity that the stimulus occurs (Latency from OOemg onset) in msec.

low-intensity SC stimulation that ended $5 \mathrm{msec}$ before the corneal stimulus (Fig. 1A). The magnitude of SC suppression was very sensitive to the timing of the SC stimulus train relative to the corneal stimulus. Starting the SC stimulus train $10 \mathrm{msec}$ after the corneal stimulus, which was just before OOemg activity should normally begin, resulted in the largest suppression (Fig. $1 C ; 10$ $\mathrm{msec})$. As the onset of the stimulus train moved ahead in time relative to the corneal stimulus (Fig. $1 B$ ), the amount of SC induced suppression declined (Fig. 1C). In contrast to the up to 45 min period of analgesia produced by PAG stimulation (Levine et al., 1991), an SC stimulus train had no significant effect on reflex blinks evoked $60 \mathrm{msec}$ after the termination of the stimulus train (Fig. $1 C ;-130 \mathrm{msec}$ ). Also consistent with the lack of PAG involvement in SC suppression of reflex blinks, a systemic injection of naloxone at concentrations sufficient to block PAG antinociceptive activity (Cazala and David, 1991; Kamei et al., 1992) failed to alter the ability of SC stimulation to suppress reflex blinks in two animals $\left(t_{(3)}=1.7\right.$, not significant). Thus, although stimulation sites for producing reflex blink suppression were in the deep layers of the SC (Basso et al., 1996), this effect did not involve activation of PAG antinociceptive centers.

Microstimulation of almost all brainstem areas receiving SC afferents suppressed trigeminal reflex blinks. For example, stimulation of the NRM with current intensities as low as $15 \mu \mathrm{A}$ readily suppressed reflex blinks (Fig. $2 A$ ). Seventy millisecond trains of stimuli significantly suppressed OOemg activity in all four rats tested (Fig. $2 B$; Kruskal-Wallis $=6.05, p<0.01$ ). The trigeminal reflex blink suppression produced by brainstem microstimulation could have occurred because the electrical stimulus 


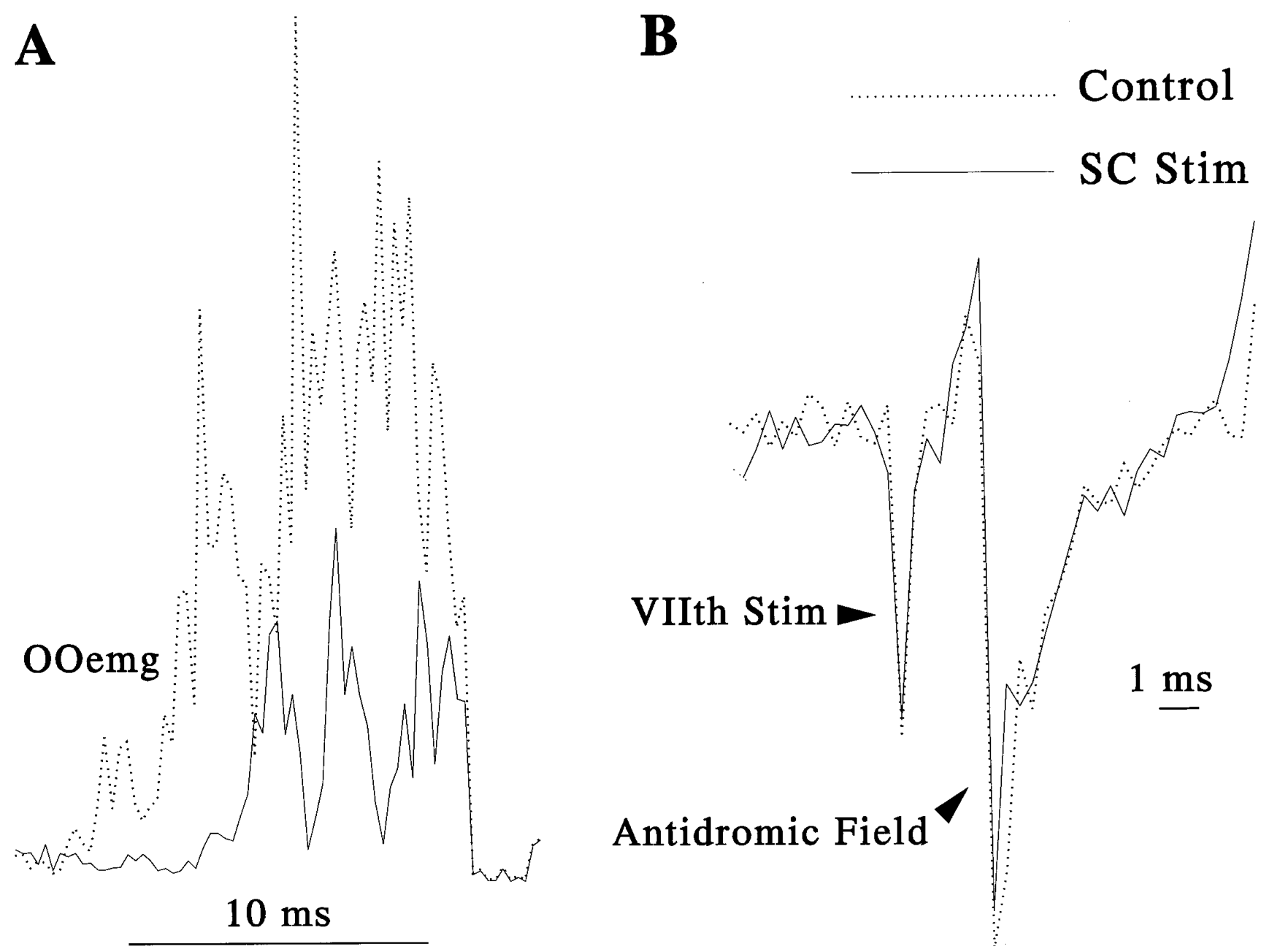

Figure 4. Effect of trains of SC microstimulation on facial nucleus antidromic field potentials. $A$, OOemg response to corneal stimulation at the intensity used to assess $\mathrm{OO}$ motoneuron excitability with (solid line) and without (dashed line) preceding, $70 \mathrm{msec}$ train of SC stimulation. B, Antidromic field potential from the $\mathrm{OO}$ subdivision of the facial nucleus evoked by stimulation of the zygomatic branch of the facial nerve with (solid line) and without (dashed line) preceding SC stimulation. Each trace is the average of 10 responses.

activated fibers of passage from the SC or because several independent pools of neurons linked the SC to trigeminal reflex blink circuits. As an initial step to establish a causal linkage, we determined the latency of OOemg suppression after a single stimulus to the SC and repeated this procedure in candidate brainstem structures.

At SC sites where trains of low-intensity, SC stimulation induced reflex blink suppression, a single pulse presented to the SC of five animals during a corneal-evoked reflex blink transiently suppressed OOemg activity (Fig. $3 A$ ). As suggested by the time course experiment (Fig. 1), the SC stimulation was more effective and reliable when it occurred during the OOemg response. Because the latency of the OOemg response to corneal stimulation varied between animals, the data from only two of these animals could be used to determine a reliable latency measure. When there was sufficient OOemg activity before the onset of the single SC pulse (i.e., at least $10 \mathrm{msec}$ ), the mean latency to the transient OOemg suppression was $5.4 \mathrm{msec}$ (Fig. 3, Table 1).

If a brainstem area links the SC to the trigeminal reflex blink circuits, stimulating that brainstem nucleus should produce suppression at a shorter latency than SC stimulation at all delays. We tested the nucleus pontis caudalis (NPc), the nucleus paragigan- tocellularis (PGi), the NRM, and the nucleus supragenualis rostral to the prepositus hypoglossi nucleus (S.Genu/PH). Trains of low-intensity stimuli presented to all of these brainstem areas suppressed reflex blinking. The NRM, however, was the only structure in which stimulation transiently suppressed OOemg with a latency shorter than SC stimulation in all four animals tested (Fig. 3B, Table 1). Other neural structures that also transiently suppressed OOemg activity with single-pulse stimulation did so at latencies that were much longer and more variable than those obtained with NRM stimulation. Single-pulse stimuli delivered to NRM also produced deeper suppression than the other structures. The mean 4.4 msec latency suppression of OOemg activity with NRM stimulation in four animals is consistent with a single synapse intervening between the SC and the NRM.

Reflex blink suppression caused by SC stimulation could occur at any one of the three neurons of the corneal reflex blink circuit: (1) A $\delta$ primary afferents; (2) spinal trigeminal second-order neurons; or (3) OO motoneurons. Previous work showed that stimulation of the NRM exerted its suppressive effects on trigeminal neuron responsiveness (Dostrovsky, 1980). If the SC suppressed reflex blinks through the NRM, then SC stimulation should not inhibit $\mathrm{OO}$ motoneuron activity but, rather, should reduce spinal 


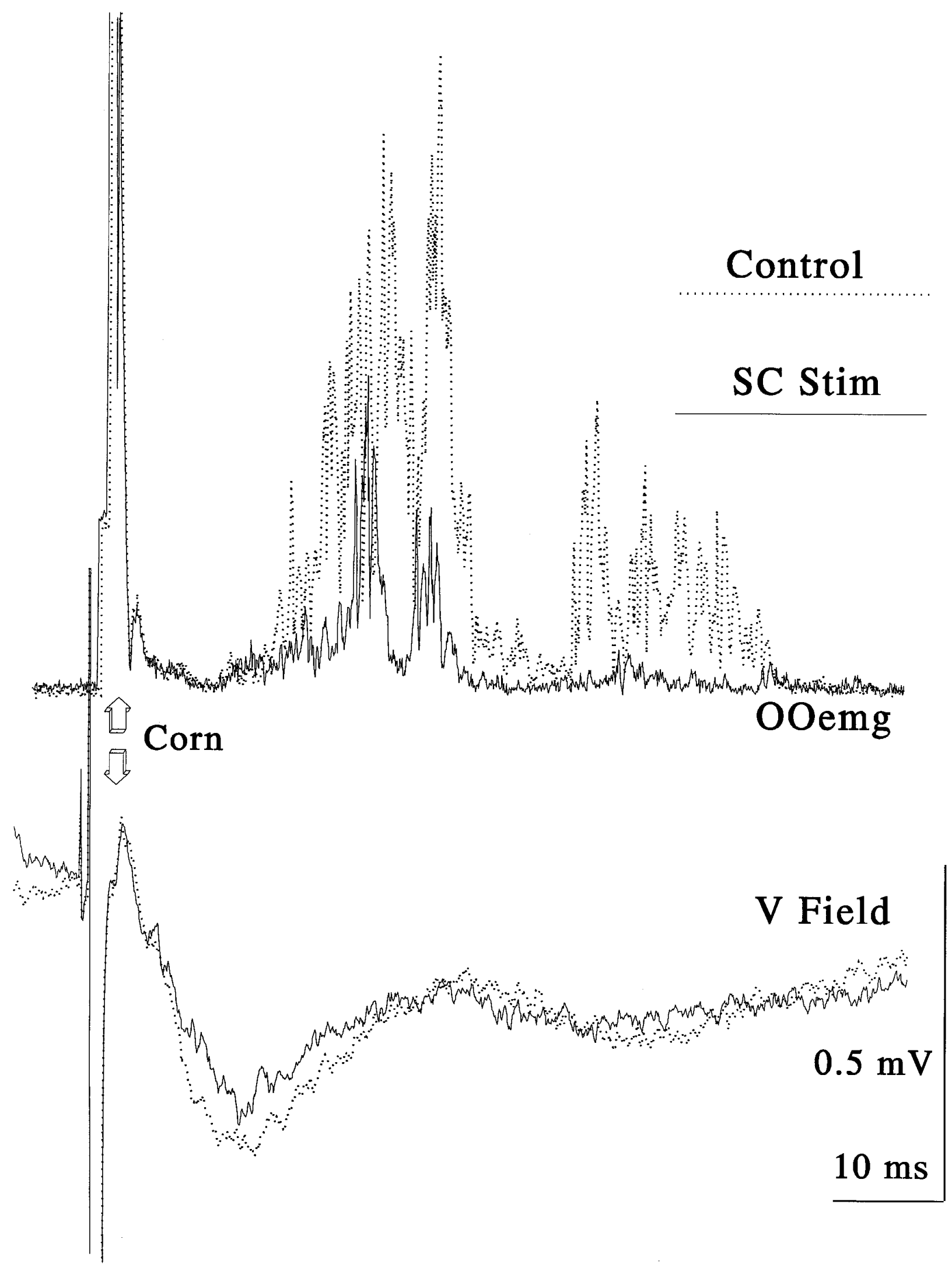

Figure 5. Effect of a train of SC stimulation on simultaneously recorded OOemg activity and trigeminal field potentials evoked by a corneal stimulus ( $\Downarrow$ Corn $)$. Top traces are superimposed orbicularis oculi emg (OOemg) responses, and bottom traces are superimposed trigeminal field potentials (V Field). Solid lines show trials with a preceding, $70 \mathrm{msec}$ train of SC stimulation (SC Stim) that terminated 5 msec before the corneal stimulus. Dotted lines show records from unstimulated trails (Control). Each trace is an average of 10 responses. 


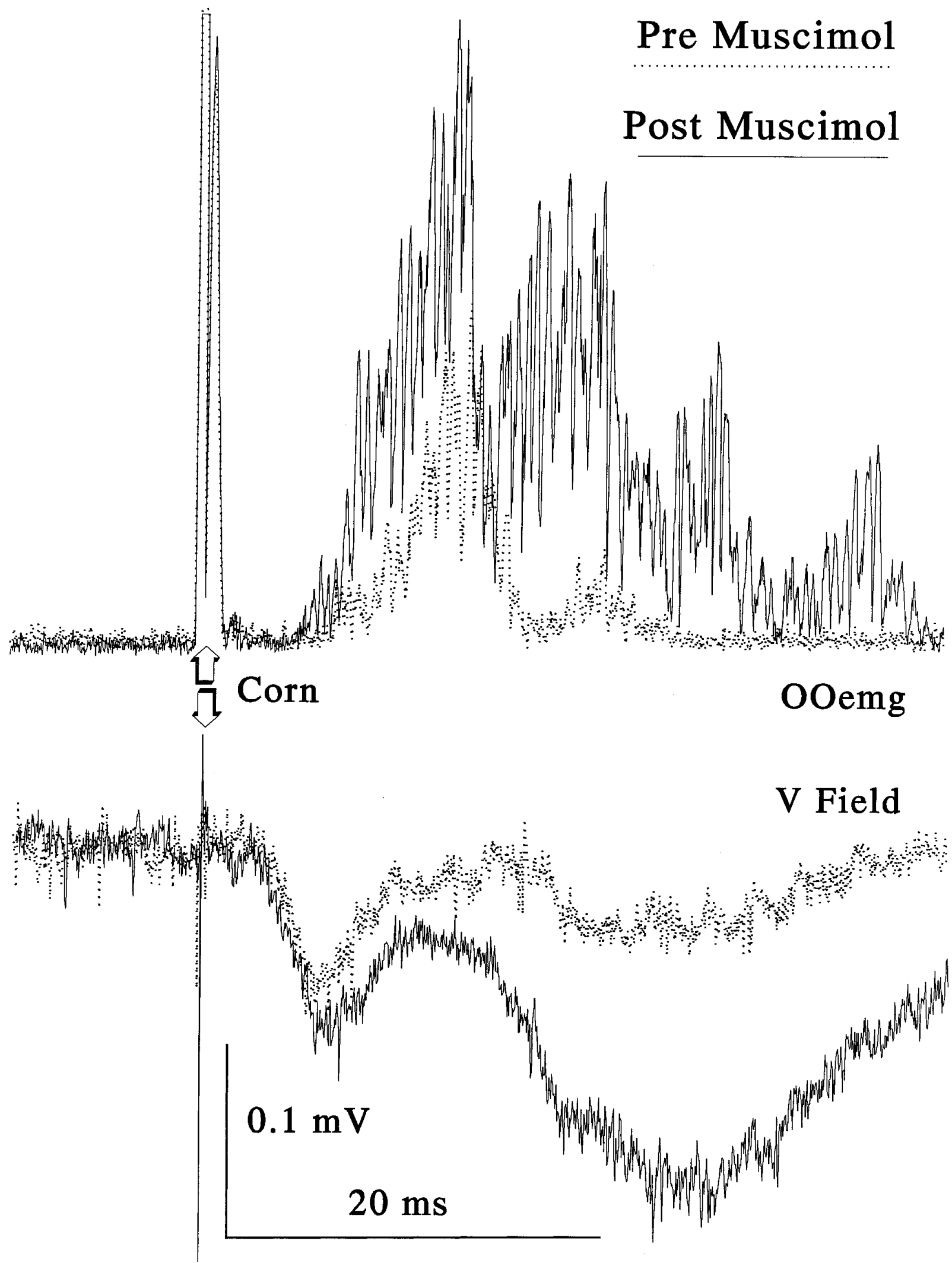

Figure 6. Effect of a microinjection of muscimol into the SC stimulation on simultaneously recorded OOemg activity and trigeminal field potentials evoked by a corneal stimulus ( Corn). Top traces are superimposed orbicularis oculi emg (OOemg) responses, and bottom traces are superimposed trigeminal field potentials (V Field). Solid lines show trials after a $0.5 \mu l$ injection of $1.0 \%$ muscimol into the contralateral SC (Post Muscimol). Dotted lines show records collected before the muscimol injection (Pre Muscimol). Each trace is an average of 12 responses. 


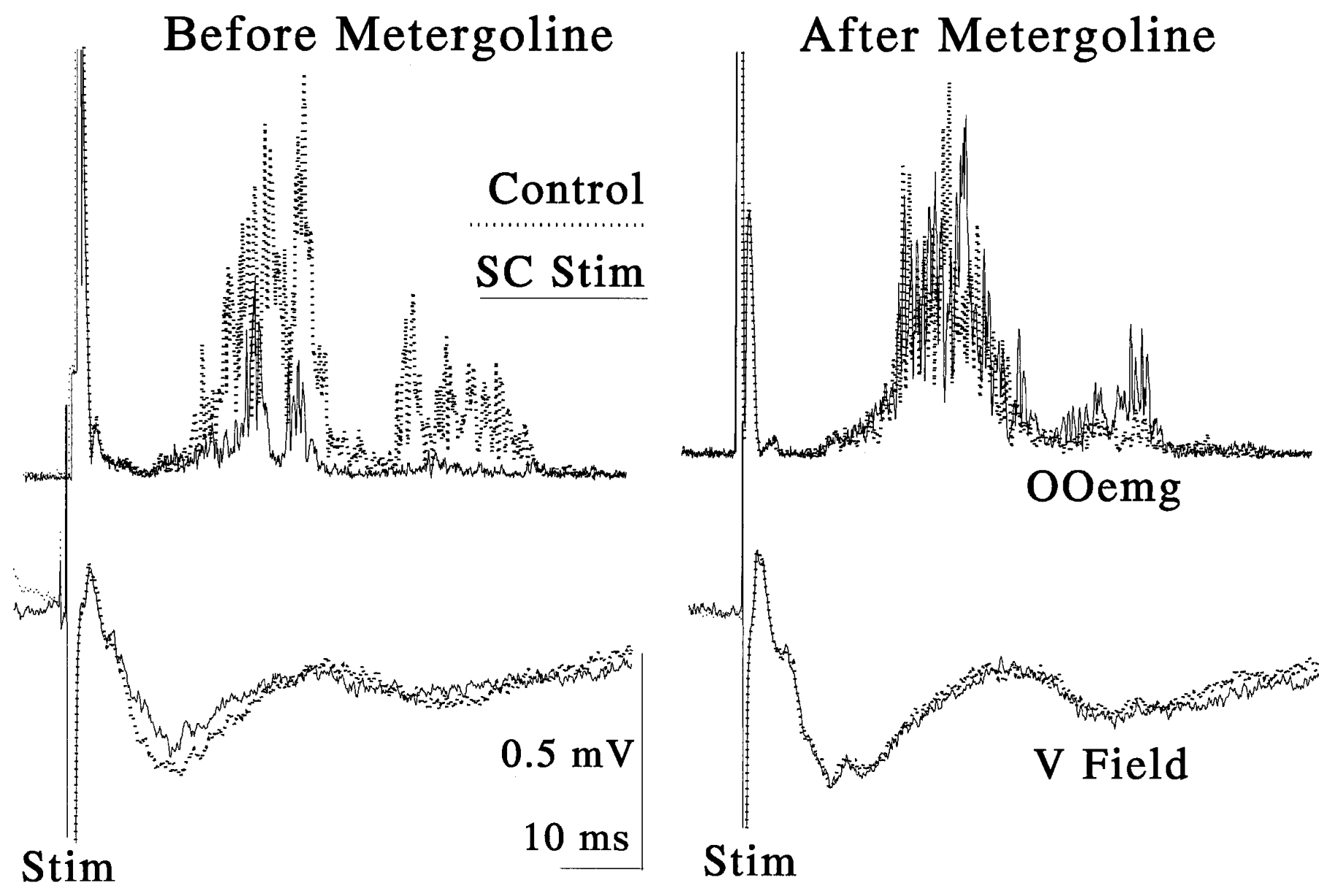

Figure 7. Systemic metergoline injections block SC suppression of orbicularis oculi (OOemg) and trigeminal field potentials (V Field) evoked by corneal stimulation (Stim). Simultaneously recorded OOemg (top traces) and trigeminal field responses (bottom traces) to a corneal stimulus with (solid line, SC Stim) and without (dotted line, Control) a preceding, $70 \mathrm{msec}$ SC stimulation that terminated $5 \mathrm{msec}$ before the corneal stimulus before (left records) and after (right records) a $6 \mathrm{mg} / \mathrm{kg}$ injection of metergoline. Each trace is an average of 10 rectified responses.

trigeminal activity. We tested whether the SC acted at the efferent limb of the corneal blink reflex by determining whether SC stimulation reduced the antidromic field potential of $\mathrm{OO}$ motoneurons. Antidromic fields recorded from $\mathrm{OO}$ motoneuron pools showed no significant change with SC stimulation (Fig. 4B) that substantially suppressed the reflex blinks (Fig. 4A). As expected from previous studies (Vidal et al., 1988; May et al., 1990), however, antidromic fields produced by vibrissal motoneurons showed a decrease in amplitude with SC stimulation (data not shown).

Unlike the case with $\mathrm{OO}$ motoneurons, SC stimulation decreased the activity of the trigeminal nucleus as well as OOemg magnitude (Fig. 5). Primary afferent fibers innervating the rat cornea arise from the ophthalmic division of the trigeminal nerve and terminate within the trigeminal nucleus at the border between the rostral caudalis subdivision and the caudal interpolaris subdivision (Marfurt and Del Toro, 1987). Because neurons in this region project to OO motoneurons (Pellegrini et al., 1995), changes in the neuronal activity in this region can significantly influence corneal evoked reflex blinks. The latency of the field in the spinal trigeminal nucleus produced by a corneal stimulus was $5.11 \pm 0.422 \mathrm{msec}$ (10 responses for each of 6 animals) and preceded OOemg activity. Presenting trains of low-intensity stimuli to the SC reduced the magnitude of the spinal trigeminal field and concomitantly suppressed the OOemg activity in 10 of the 11 animals tested. The mean difference in the field potential amplitude with and without SC stimulation was $0.187 \mathrm{mV}$ (sign test: $p<$ $0.05)$. Between animals, the amount of trigeminal field suppression produced by SC stimulation varied between 5 and $88 \%$, and the amount of blink suppression varied between 5 and $94 \%$. Consistent with the SC activation suppressing the afferent limb of the reflex, inhibiting SC activity with a $0.5 \mu \mathrm{l}(1.0 \%)$ muscimol injection into the SC increased the size of the trigeminal field and blink amplitude (Fig. 6) (Basso et al., 1996).

Stimulation of the NRM causes the release of serotonin in the trigeminal nucleus (Shibutani, 1990). If the SC excited the NRM to suppress reflex blinking, then blocking serotonin receptors should prevent SC stimulation from suppressing reflex blinks. The ability of SC stimulation to reduce trigeminal field potentials evoked by corneal stimulation and reflex blinks was examined before and after a systemic injection of $6 \mathrm{mg} / \mathrm{kg}$ metergoline, a serotonin receptor blocker (Fig. 7) (Middlemiss and Trickleybank, 1992). Blocking serotonin receptors prevented SC stimulation from decreasing the trigeminal field potential and subsequent OOemg activity. The median decrease in corneally evoked trigeminal field amplitude caused by SC stimulation was $10 \%$. Systemic injection of $6 \mathrm{mg} / \mathrm{kg}$ metergoline in four animals reduced the efficacy of SC stimulation on the trigeminal field by $46 \%$. Simi- 


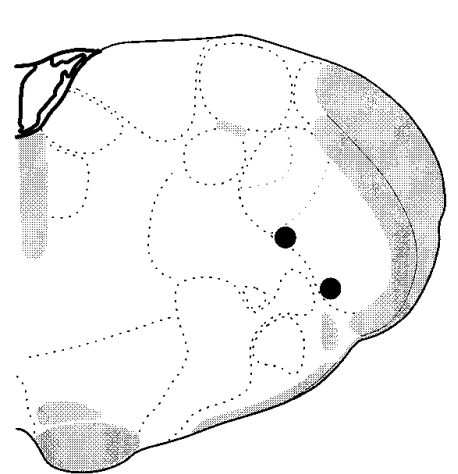

$-11.75$

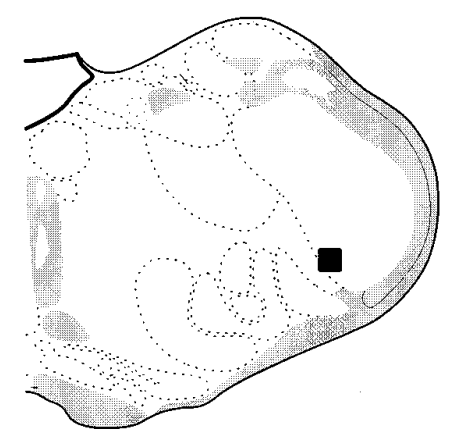

$-13.28$

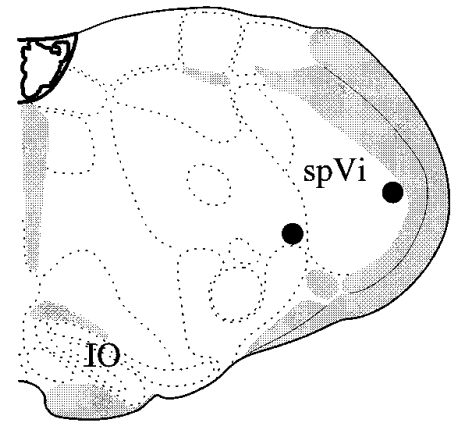

$-12.50$
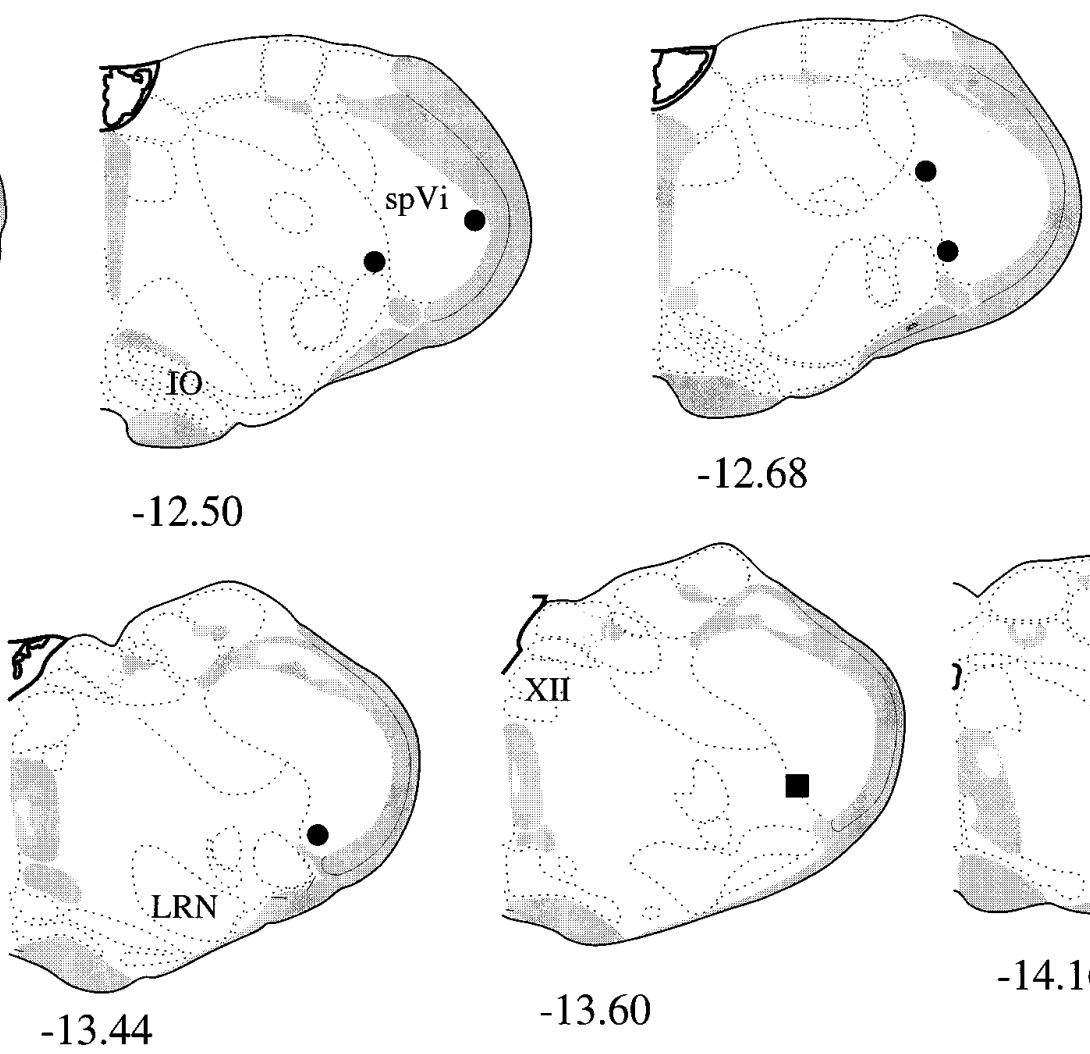

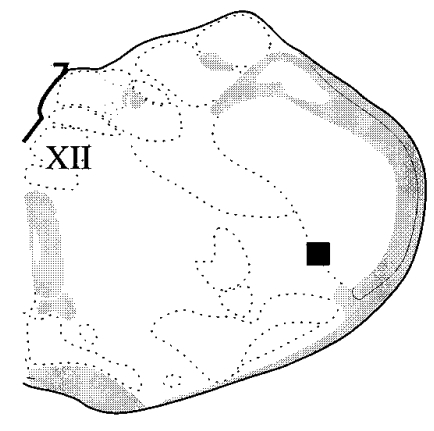

$-13.60$

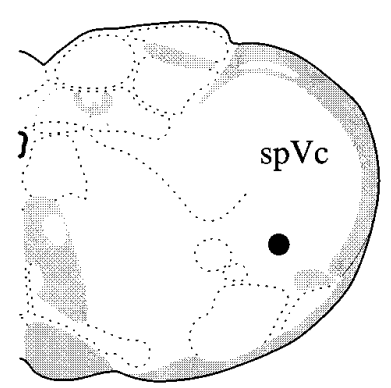

$-14.16$

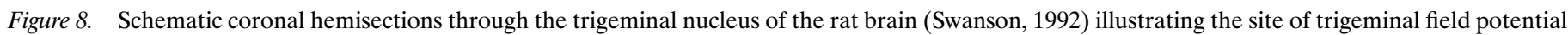

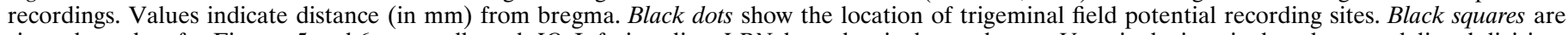

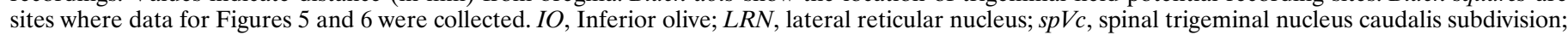
$s p V i$, spinal trigeminal nucleus interpolaris subdivision; XII, hypoglossal nucleus.

larly, blocking serotonin receptors reduced SC suppression of trigeminal reflex blinks by $73 \%\left(t_{(3)}=-4.03, p<0.05\right)$. The difference in suppression of the trigeminal field and the OOemg suggested that the electrodes in the spinal trigeminal nucleus were not always optimally placed to record corneally evoked trigeminal field potentials (Fig. 8). Systemic injection of the vehicle ascorbic acid in two animals had no effect on the SC induced trigeminal field suppression or reflex blink suppression $\left(t_{(1)}=-1.20\right.$, not significant).

The ability of SC stimulation to suppress reflex blinking was examined before and after reversible inactivation of the NRM with microinjections of muscimol in eight animals (Fig. 9; 1 animal $0.5 \mu \mathrm{l} ; 7$ animals $50-100 \mathrm{nl} ; 1.0 \%$ ). At sites where trains of NRM stimulation suppressed reflex blinking (6 rats), a microinjection of muscimol significantly reduced the ability of SC stimulation to suppress blinking (Fig. $9 B ; t_{(5)}=-2.65, p<0.05$ ). The average reduction in reflex blink amplitude produced by SC stimulation was $72 \%$. After inactivation of the NRM, however, the average reflex blink suppression produced by SC stimulation was only $33 \%$ (Fig. 9B). Histological analysis revealed all electrode placements that altered SC stimulation-induced suppression of reflex blinks were within the NRM (Fig. 10). Two animals survived long enough to recover from the NRM inactivation. In these rats, suppression of reflex blinks by SC stimulation returned to normal within 90 to $120 \mathrm{~min}$. In the two animals with no measurable effect of muscimol injection, histological analysis revealed that the electrodes were located outside of the NRM (Fig. 10).

\section{DISCUSSION}

Changes in the level of SC activity dramatically modify reflex blink magnitude. Inhibition of the SC produced marked reflex blink hyperexcitability, whereas activating the SC reduced trigeminal reflex blink amplitude (Basso et al., 1996). These data support the hypothesis that the SC modulates tonically active neurons that inhibit the reflex blink circuit. The present results demonstrate that the NRM links the SC to trigeminal reflex blink circuits. That these neurons could fulfill this role is supported further by anatomical evidence demonstrating a projection from the SC to the NRM (Carlton et al., 1983) and physiological evidence demonstrating that serotonergic NRM neurons are tonically active (Fornal et al., 1985) and that NRM neurons excited by SC stimulation are tonically active (our unpublished observations). Not all serotonergic cells within the NRM are nociceptive-specific (Fornal et al., 1985), and not all serotonergic effects are opioid-sensitive (Satoh et al., 1980; Gebhardt et al., 1983a,b). The present results are consistent with these data because they demonstrate that the effects of SC stimulation do not result from directly or indirectly activating descending antinociceptive mechanisms originating in the PAG. The duration of SC-induced reflex blink suppression is much shorter than the stimulus-induced analgesia that results from PAG stimulation (Levine et al., 1991), and reflex blink suppression by SC stimulation is unaffected by an opioid antagonist, nalaxone.

The SC could suppress the corneal blink reflex by inhibiting the primary afferents, the trigeminal or reticular interneurons, or the 

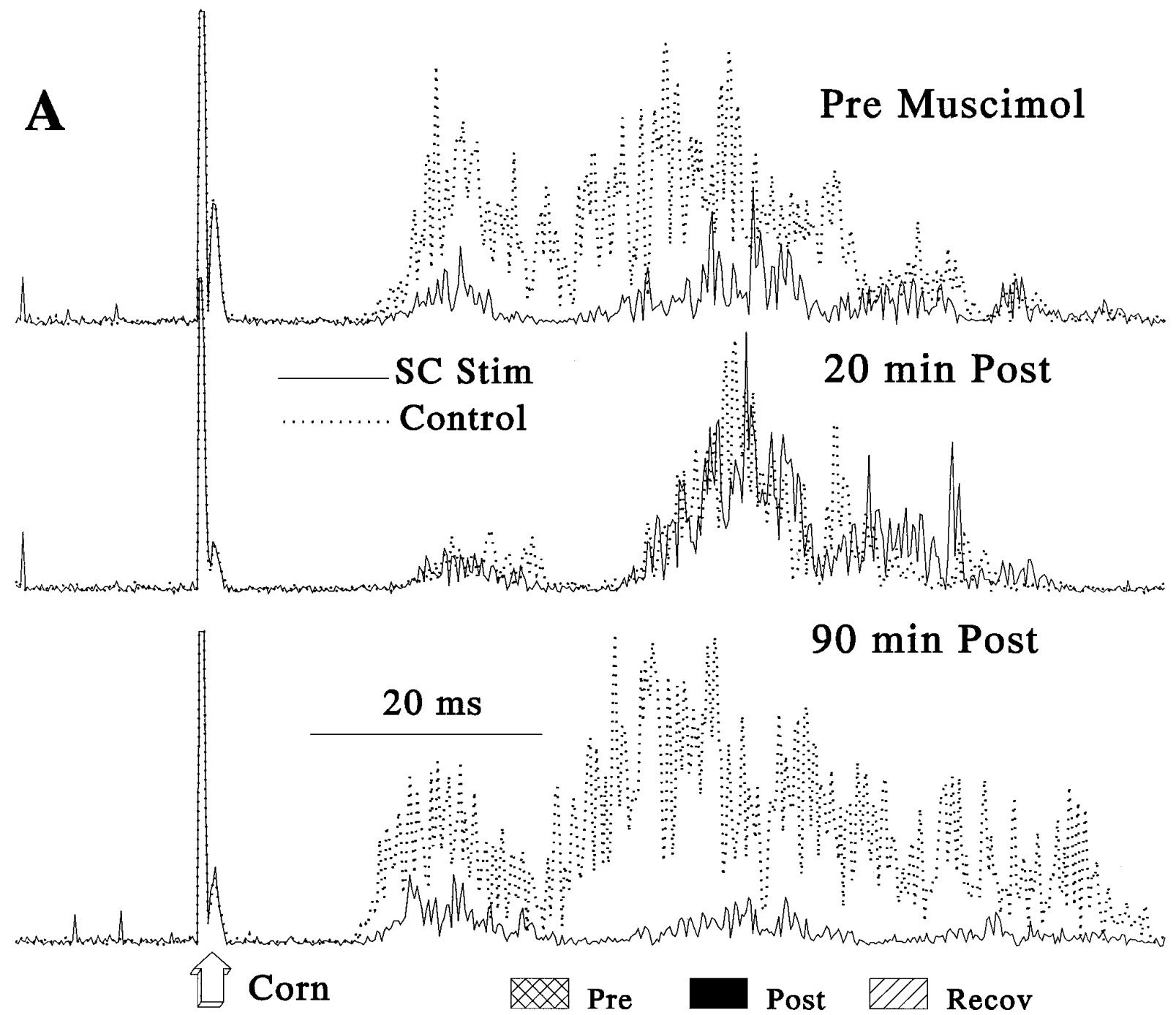

B

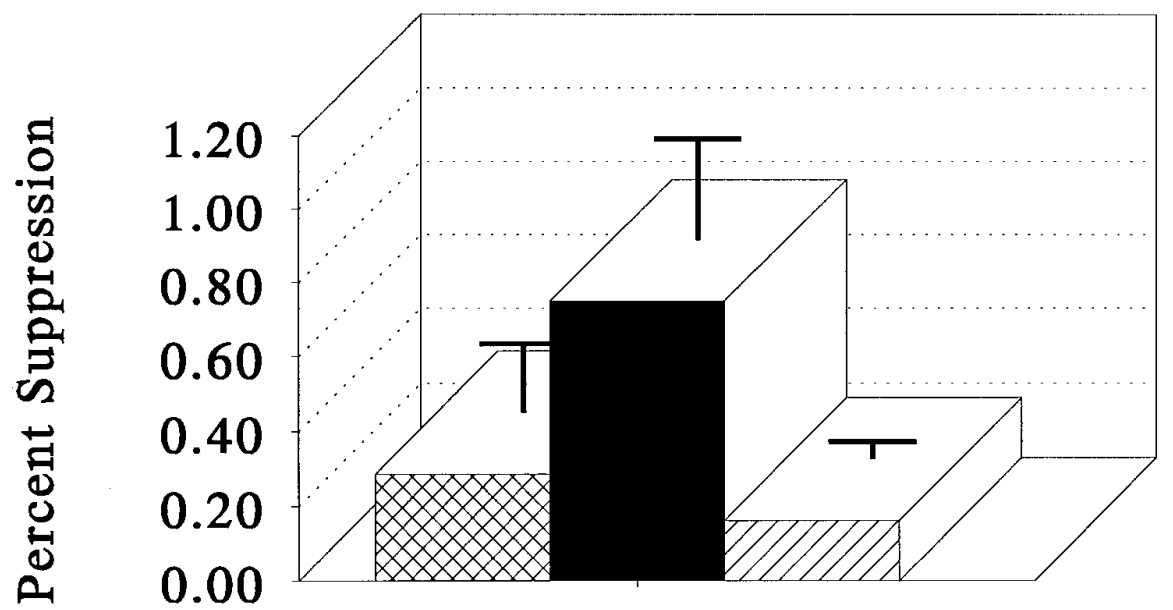

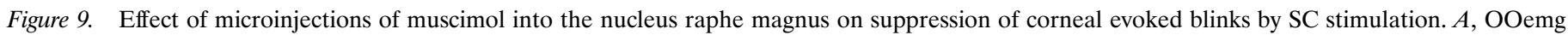

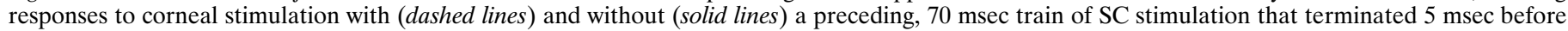

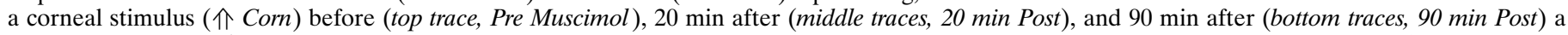

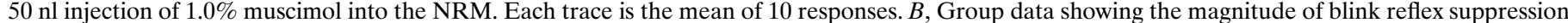

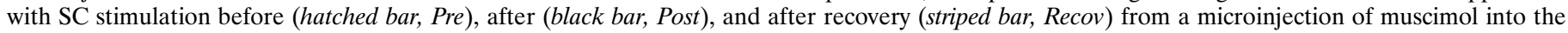

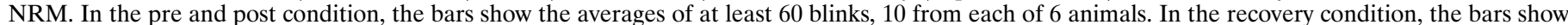
averages of at least 20 blinks, 10 from each of two of these 6 animals. Error bars are SEM. 


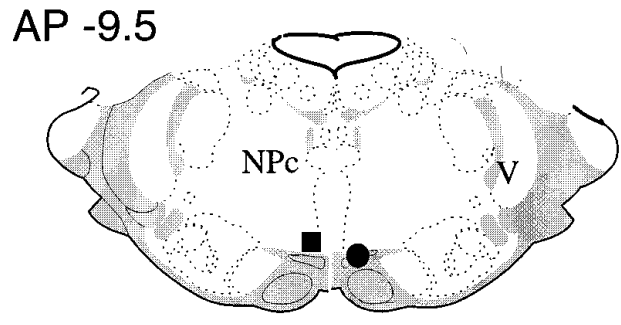

AP -10.35
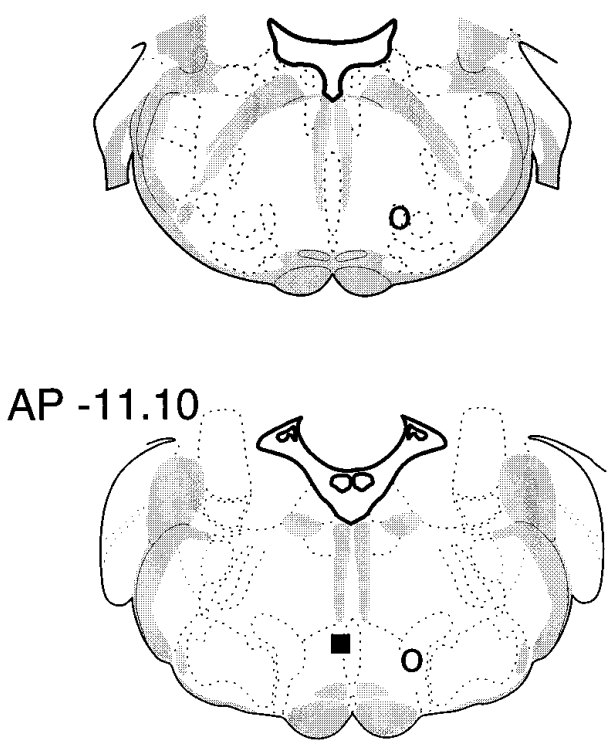
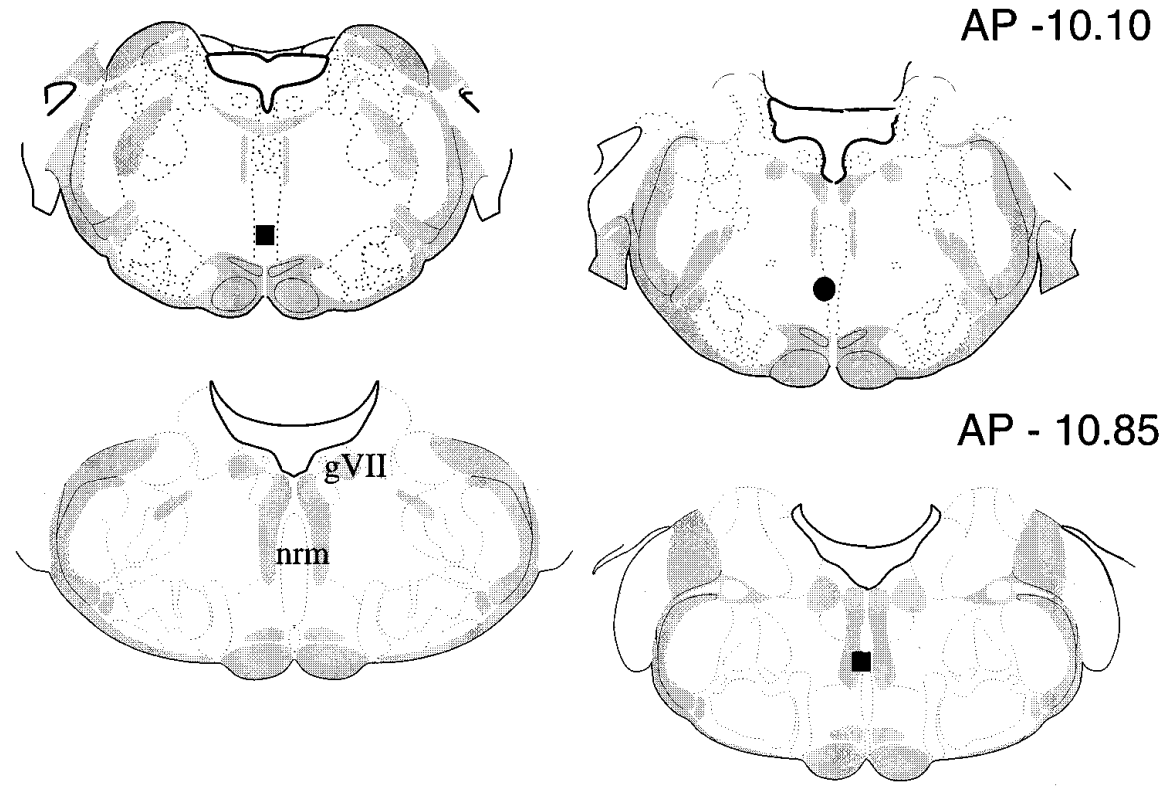

- 0 - 10 minutes

- $30-45$ minutes

- No Effect

Figure 10. Location of muscimol injections in the nucleus raphe magnus experiments. Black squares show injection sites that reduced SC suppression of trigeminal reflex blinks within 10 min. Filled circles show injection sites where reduction of SC blink suppression occurred between 30 and 45 min after the injection. The open circles identify ineffective injection sites. $g V I I$, Genu of the facial nerve; $N P c$, nucleus pontis caudalis; $N R M$, nucleus raphe magnus.

OO motoneurons that form the minimal corneal reflex blink circuit. Two lines of evidence argue against the SC acting to inhibit $\mathrm{OO}$ motoneuron activity. First, SC stimulation did not reliably alter the excitability of OO motoneurons (Fig. 4). Second, the facial motoneurons that receive disynaptic inhibition from SC stimulation tend to innervate auricular muscles (Vidal et al., 1988; May et al., 1990). The evidence supporting the hypothesis that SC stimulation reduces the activity of second-order trigeminal neurons in response to corneal stimulation is compelling. SC stimulation consistently decreases second-order trigeminal fields evoked by corneal stimulation (Fig. 5). This decrease could have resulted from primary afferent depolarization (PAD) of trigeminal afferent terminals or direct inhibition of second-order trigeminal neurons. There are numerous studies demonstrating PAD in the trigeminal system (Nakumura and Wu, 1970; Goldberg, 1972; Goldberg and Browne, 1974; Yu and Avery, 1974; Browne and Goldberg, 1978). Previous studies also show that the proposed link between the SC and reflex blinks circuits, the NRM, reduces trigeminal neuron responsiveness to primary afferents (Dostrovsky, 1980).

The current experiments provide strong evidence that the NRM links the SC to the blink circuit. Stimulation of the SC suppresses OOemg activity in $5.4 \mathrm{msec}$, whereas microstimulation of the NRM suppresses OOemg activity in $4.4 \mathrm{msec}$. The $1 \mathrm{msec}$ latency difference is consistent with a monosynaptic projection from the $\mathrm{SC}$ to the NRM. Because the contralateral descending collicular efferent system originating in the rostral and lateral SC projects to the pontine and medullary raphe nuclei (rat: Redgrave et al., 1987; cat: Kawamura et al., 1974), and specifically to the NRM (Carlton et al., 1983), anatomical evidence supports this physiological result. Blocking serotonin receptors prevents the SC from suppressing reflex blinks. This is consistent with NRM linking SC and blink circuits because NRM projections to the trigeminal complex are serotonergic and NRM stimulation releases serotonin in the trigeminal complex (Shibutani, 1990) (for review, see Jacobs and Azmitia, 1992). Moreover, NRM stimulation suppresses corneal-related neurons in the trigeminal nucleus (Lovick and Wolstencroft, 1979). Thus, serotonin antagonists should block the effect of SC suppression of blink reflexes if the NRM mediates the effect. Finally, temporarily inactivating NRM with muscimol blocked the effect of SC stimulation on the blink reflex (Fig. 9).

The present results suggest that reflex blink amplitude correlates inversely with NRM activity and serotonin release in the trigeminal nucleus. The modulation of reflex blink excitability during REM sleep supports this idea. For example, NRM neurons exhibit a decrease to a near cessation of their spontaneous tonic activity during REM sleep (Auerbach et al., 1985; Jacobs and Azmitia, 1992), which should increase reflex blink amplitude. As predicted, the long-latency, R2 component of the supraorbitally evoked reflex blink in humans increases in duration during REM sleep relative to blinks evoked when the subjects are awake (Kimura and Harada, 1972). The role of serotonergic neurons in 


\section{Basal Ganglia Modulation of Reflex Blinks}

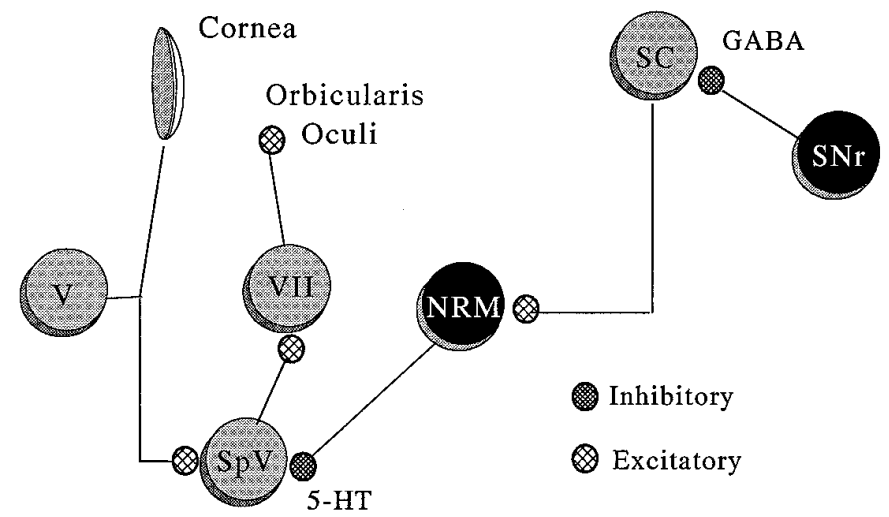

Figure 11. Circuit linking the basal ganglia and the trigeminal reflex blink circuit. $N R M$, Nucleus raphe magnus; $S C$, superior colliculus; $S N r$, substantia nigra pars reticulata; $S p V$, spinal trigeminal nucleus; $V$, trigeminal ganglion; VII, facial motoneurons.

the NRM as well as other raphe nuclei typically involves both a motor facilitation and a sensory suppression. Jacobs and Fornal (1992) hypothesize that the suppression of sensory information occurs as a result of the activation of serotonergic cells involved in motor behavior. Consistent with this, voluntary arm movements can suppress reflex blinks (Sanes, 1984).

\section{An explanation of reflex blink hyperexcitability in Parkinson's disease}

The present experiments combined with those of the previous study (Basso et al., 1996) argue that the basal ganglia can regulate reflex blink excitability by altering the inhibitory drive from the $\mathrm{SNr}$ to the SC. In turn, the level of SC activation modulates the activity of NRM neurons that tonically inhibit spinal trigeminal nucleus responsiveness, the afferent limb of the reflex blink (Fig. 11). The dopamine depletion of Parkinson's disease leads to an increase in $\mathrm{SNr}$ activity. This increased inhibitory output decreases the activity of lateral and rostral SC neurons. The loss of the SC excitatory drive on continuously active NRM neurons removes some of the tonic inhibition of trigeminal neuronal responsiveness, which leads to reflex blink hyperexcitability. Conversely, Huntington's disease, which causes a decrease in $\mathrm{SNr}$ inhibition of the SC, leads to reflex blink hypoexcitability. Thus, the tonic level of BG output modulates trigeminal reflex blink excitability.

It is possible that, in addition to modulating tonic reflex blink magnitude, the BG modifies reflex amplitude phasically. McHaffie et al. (1989) suggested that noxious facial stimuli transiently increase the output of the BG, which suppresses orienting behavior to allow unopposed withdrawal or defensive behaviors. In addition to suppressing orienting behavior, the current data show that the increased BG output increases the sensitivity of cutaneous reflexes to enhance the protective function of trigeminal reflex blinks.

\section{REFERENCES}

Auerbach S, Fornal C, Jacobs B (1985) Response of serotonin containing neurons in nucleus raphe magnus to morphine, noxious stimuli, and periaqueductal gray stimulation in freely moving cats. Exp Neurol 88:609-628.

Basso MA, Strecker RE, Evinger C (1993) Midbrain 6-hydroxdopamine lesions modulate blink reflex excitability. Exp Brain Res 94:88-96.
Basso MA, Powers AS, Evinger C (1996) An explanation for blink reflex hyperexcitability in Parkinson's disease. I. Superior colliculus. J Neurosci 16:7308-7317.

Bronstein AM, Kennard C (1985) Predictive ocular motor control in Parkinson's disease. Brain 108:925-940.

Browne PA, Goldberg LJ (1978) Direct evidence of presynaptic inhibition of a trigeminal reflex. Brain Res 143:369-372.

Büttner-Ennever JA, Cohen B, Pause M, Fries W (1988) Raphe nucleus of the pons containing omnipause neurons of the oculomotor system in the monkey, and its homologue in man. J Comp Neurol 267:307-321.

Carlton S, Leichnetz G, Young E, Mayer D (1983) Supramedullary afferents of the nucleus raphe magnus in the rat: a study using the transcannula HRP gel and autoradiographic techniques. J Comp Neurol 214:43-58.

Cazala P, David V (1991) Differential effects of naloxone on approach and escape response induced by electrical stimulation of the lateral hypothalamus or the mesencephalic central gray area in mice. Pharmacol Biochem Behav 40:323-327.

Dean P, Redgrave P, Sahibzada N, Tsuji K (1986) Head and body movements produced by electrical stimulation of superior colliculus in rats: effects of interruption of crossed tectoreticulospinal pathway. Neuroscience 19:367-380.

Dean P, Mitchell IJ, Redgrave P (1988a) Contralateral head movements produced by microinjection of glutamate into superior colliculus of rats: evidence for mediation by multiple output pathways. Neuroscience 24:491-500.

Dean P, Mitchell IJ, Redgrave P (1988b) Responses resembling avoidance behaviour produced by microinjection of glutamate into superior colliculus of rats. Neuroscience 24:501-510.

Dean P, Redgrave P, Westby GWM (1989) Event or emergency? Two response systems in the mammalian superior colliculus. Trends Neurosci 12:137-147.

Dostrovsky JO (1980) Raphe and periaqueductal gray induced suppression of non-nociceptive neuronal responses in the dorsal column nuclei and trigeminal sub-nucleus caudalis. Brain Res 200:184-189.

Dostrovsky JO, Hu JW, Sessle BJ, Sumino R (1982) Stimulation sites in the periaqueductal gray, nucleus raphe magnus and adjacent regions effective in suppression oral-facial reflexes. Brain Res 252:287-297.

Evinger C, Kaneko CRS, Fuchs AF (1982) Activity of omnipause neurons in alert cats during saccadic eye movements and visual stimuli. J Neurophysiol 47:827-844.

Fornal CA, Auerbach S, Jacobs BL (1985) Activity of serotonin containing neurons in the nucleus raphe magnus in freely moving cats. Exp Neurol 88:590-608.

Fuchs AF, Ling L, Kaneko CRS, King WM, Usher D (1991) The timing of the response of brainstem omnipause neurons relative to saccadic eye movements in rhesus monkeys. Soc Neurosci Abstr 17:462.

Gebhardt GF, Sandkühler J, Thalhammer JG, Zimmerman M (1983a) Quantitative comparison of inhibition in spinal cord of nociceptive information by stimulation in periaqueductal gray or nucleus raphe magnus of the cat. J Neurophysiol 50:1433-1445.

Gebhardt GF, Sandkühler J, Thalhammer JG, Zimmerman M (1983b) Inhibition of spinal nociceptive information by stimulation in midbrain of the cat is blocked by lidocaine microinjected in nucleus raphe magnus and medullary reticular formation. J Neurophysiol 50:1446-1459.

Goldberg LJ (1972) Excitatory and inhibitory effects of lingual nerve stimulation of reflexes controlling the activity of masseteric motoneurons. Brain Res 39:95-108.

Goldberg LJ, Browne PA (1974) Differences in the excitability of two populations of trigeminal primary afferent central terminals. Brain Res 77:195-209.

Grantyn R (1988) Gaze control through the superior colliculus. In: Neuroanatomy of the oculomotor system (Büttner-Ennever JA, ed), pp 273-333. Amsterdam: Elsevier.

Jacobs BL, Azmitia EC (1992) Structure and function of the brain serotonin system. Physiol Rev 72:165-229.

Jacobs BL, Fornal CA (1992) 5-HT and motor control: a hypothesis. Trends Neurosci 16:346-352.

Jayaraman A, Batton RRIII, Carpenter M (1977) Nigrotectal projections in the monkey: an autoradiographic study. Brain Res 135:147-152.

Kamei J, Aoki T, Kasuya Y (1992) Periaqueductal gray matter stimulation-produced analgesia in diabetic rats. Neurosci Lett 142:13-16. 
Kawamura K, Brodal A, Hoddevik G (1974) The projection of the superior colliculus onto the reticular formation of the brainstem an experimental anatomical study in the cat. Exp Brain Res 19:1-19.

Kimura J (1973a) Disorders of interneurons in Parkinson's disease. Brain 96:87-96.

Kimura J, Harada O (1972) Excitability of the orbicularis oculi reflex in all night sleep: its suppression in non-rapid eye movement and recovery in rapid eye movement sleep. Electroencephalogr Clin Neurophysiol 33:369-377.

Levine RL, Morgan MM, Cannon JT, Liebsekind JC (1991) Stimulation of the periaqueductal gray matter of the rat produces a preferential ipsilateral antinocioception. Brain Res 567:140-144.

Lovick TA, Wolstencroft JH (1979) Inhibitory effects of nucleus raphe magnus on neuronal responses in the spinal trigeminal nucleus compared with non-nociceptive inputs. Pain 7:135-145.

Lu SM, Breznen B, Basso MA, Evinger C, Gnadt J (1994) Effects of superior colliculus stimulation on the blink reflex in monkeys. Soc Neurosci Abstr 574:1.

Lueck CJ, Tanyeri S, Crawford TJ, Henderson L, Kennard C (1990) Antisaccades and remembered saccades in Parkinson's disease. J Neurol Neurosurg Psychiatry 53:284-288.

Marfurt CF, Del Toro DR (1987) Corneal sensory pathway in the rat: a horseradish peroxidase tracing study. J Comp Neurol 261:45-459.

May PJ, Hall WC (1984) Relationship between the nigrotectal pathway and the cells of origin of the predorsal bundle. J Comp Neurol 226:357-376.

May PJ, Hall WC (1986) The sources of the nigrotectal pathway. Neuroscience 19:159-180.

May PJ, Vidal PP, Baker R (1990) Synaptic organization of tecto-facial pathways in the cat. II. synaptic potentials following midbrain tegmentum stimulation. J Neurophysiol 64:381-402.

Mays LE, Morrisse DW (1994) Activity of pontine omnipause neurons during eye blinks. Soc Neurosci Abstr 20:574.

McHaffie JG, Kao C, Stein BE (1989) Nociceptive neurons in rat superior colliculus: response properties, topography, and functional implications. J Neurophysiol 62:510-525.

Middlemiss DN, Trickleybank MD (1992) Centrally active 5HT receptor agonists and antagonists. Neurosci Biobehav Rev 16:75-82.

Mitchell IJ, Dean P, Redgrave P (1988) The projection from the superior colliculus to the cuneiform area in the rat. Exp Brain Res 72:626-639.

Müller CH, Wenger S, Fertl L, Auff E (1994) Initiation of visual-guided random saccades and remembered saccades in Parkinsonian patients with severe motor-fluctuations. J Neural Transm 7:101-108.

Nakamura Y, Wu CY (1970) Presynaptic inhibition of jaw opening reflex by high threshold afferents from the masseter muscle of the cat. Brain Res 23:193-211.

Paré M, Guitton D (1994) The fixation area of the cat superior colliculus: effects of electrical stimulation and direct connection with brainstem omnipause neurons. Exp Brain Res 101:109-122.

Pellegrini JJ, Horn AKE, Evinger C (1995) The trigeminally evoked blink reflex. I. Neuronal circuits. Exp Brain Res 107:166-180.

Raybourn MS, Keller EL (1977) Colliculoreticular organization in primate oculomotor system. J Neurophysiol 40:861-878.

Rascol O, Clanet M, Montastruc JL, Simonetta M, Soulvier-Esteve MJ, Doyon B, Rascol A (1989) Abnormal ocular movements in Parkinson's disease. Brain 112:1193-1214.

Redgrave P, Dean P, Mitchell IJ, Odekunle A, Clarke A (1988) The projection from superior colliculus to the cuneiform nucleus in the rat. Exp Brain Res 72:611-625.

Redgrave P, Mitchell IJ, Dean P (1987) Descending projections from the superior colliculus in rat: a study using orthograde transport of wheatgerm-agglutinin conjugated horseradish peroxidase. Exp Brain Res 68:147-167.

Sanes JN (1984) Voluntary movement and excitability of cutaneous reflexes. J Psychophysiol 21:653-664.

Satoh M, Akaike A, Nakazawa T, Takagi H (1980) Evidence for involvement of separate mechanisms in the production of analgesia by electrical stimulation of the nucleus reticularis paragigantocellularis and the nucleus raphe magnus in the rat. Brain Res 194:525-529.

Shallert T, Petrie BF, Whishaw IQ (1989) Neonatal dopamine depletion: spared and unspared sensorimotor and attentional disorders and effects of further depletion in adulthood. J Psychobiol 17:386-396.

Shibutani T (1990) Mechanism of modulation of pain transmission at the subnucleus caudalis of the trigeminal sensory nuclear complex in rabbits. J Osaka Univ Dent Soc 35:594-608.

Swanson LW (1992) Brain maps: structure of the rat brain. Amsterdam: Elsevier.

Thomas RC, Wilson VJ (1965) Precise localization of renshaw cells with a new marking technique. Nature 206:211-213.

Vidal PP, May PJ, Baker R (1988) Synaptic organization of tecto-facial pathways in the cat. I. Synaptic potentials following collicular stimulation. J Neurophysiol 60:769-797.

White OB, Saint-Cyr JA, Tomlinson RD, Sharpe JA (1983) Ocular motor deficits in Parkinson's disease. II. Control of the saccadic and smooth pursuit systems. Brain 106:571-587.

Yu HH, Avery JK (1974) Primary afferent depolarization: direct evidence in the trigeminal system. Brain Res 75:328-333. 